\title{
A CONTINUUM BRIGHT POINT AT THE PENUMBRAL EDGE
}

\author{
(Research Note)
}

H. ZIRIN and R. L. MOORE

Big Bear Solar observatory, California Institute of Technology, Hale Observatories, Pasadena, Calif. 91125, U.S.A.

(Received 25 January, 1980)

\begin{abstract}
A small continuum bright point, observed at the outer edge of the penumbra of a small spot in a large complex spot group, is related to an occurrence beneath the Sun's surface. The characteristics of the point appear to be unique, and the name 'penumbra-periphery bright point' is proposed.
\end{abstract}

Bray and Loughhead have discussed the development of penumbral bright points; the various examples given in their Figure 3.11 are all in the penumbra, usually on the penumbra-umbra boundary. We have observed short-lived photospheric brightenings of similar size in association with intense flares, usually in the 3835 band (Zirin, 1980).

Our present addition to the zoo of solar features was not connected with a flare; nor was it observed in the penumbra, but at the outer edge of the penumbra of a small spot in a large, complex spot group. It was observed on September 20, 1979 at $\mathrm{Big}$ Bear simultaneously in $\lambda 3835, \mathrm{D}_{3}, \mathrm{H} \alpha$, and $\mathrm{H} \alpha-0.7 \AA$ and with a wavelength scan, $\mathrm{H} \alpha-1.0 \AA$ to $\mathrm{H} \alpha 1.0 \AA$, once per hour. Frames in $3835, \mathrm{D}_{3} \mathrm{H} \alpha$, and $\mathrm{H} \alpha-1.0 \AA$ are shown in Figure 1. The point is about equally bright in 3835 (a band $60 \AA \AA$ wide), and $\mathrm{D}_{3}$, barely visible in $\mathrm{H} \alpha-1.0$, and completely obscured in centerline. That this is not an instrumental effect was demonstrated by its presence in at least two wavelengths for about a thousand frames, moving with the solar image.

The point became visible around 18:03, and brightened at 19:45 UT, remaining bright at least until 23:30, when it was lost in the late afternoon seeing. It seemed to peak around 22:00 UT, but the measured brightness is sensitive to seeing. The bright area was $2.5^{\prime \prime}$ long, and less than $2^{\prime \prime}$ wide. It was faintly visible in emission at $\mathrm{H} \alpha$ $-0.7 \AA$ during most of its life.

A broad and sheared filament covered the point in $\mathrm{H} \alpha$ centerline, the extension of a long filament to the east. This shows that the bright point was near a magnetic inversion line, but both the structure of the filament and BBSO magnetograms show that the field gradient was not particularly strong there. The sunspot next to which the bright point occurred was really three little spots which emerged the day before and disappeared on the succeeding day. There was considerable magnetic field change in the region, not particularly confined to this spot. 


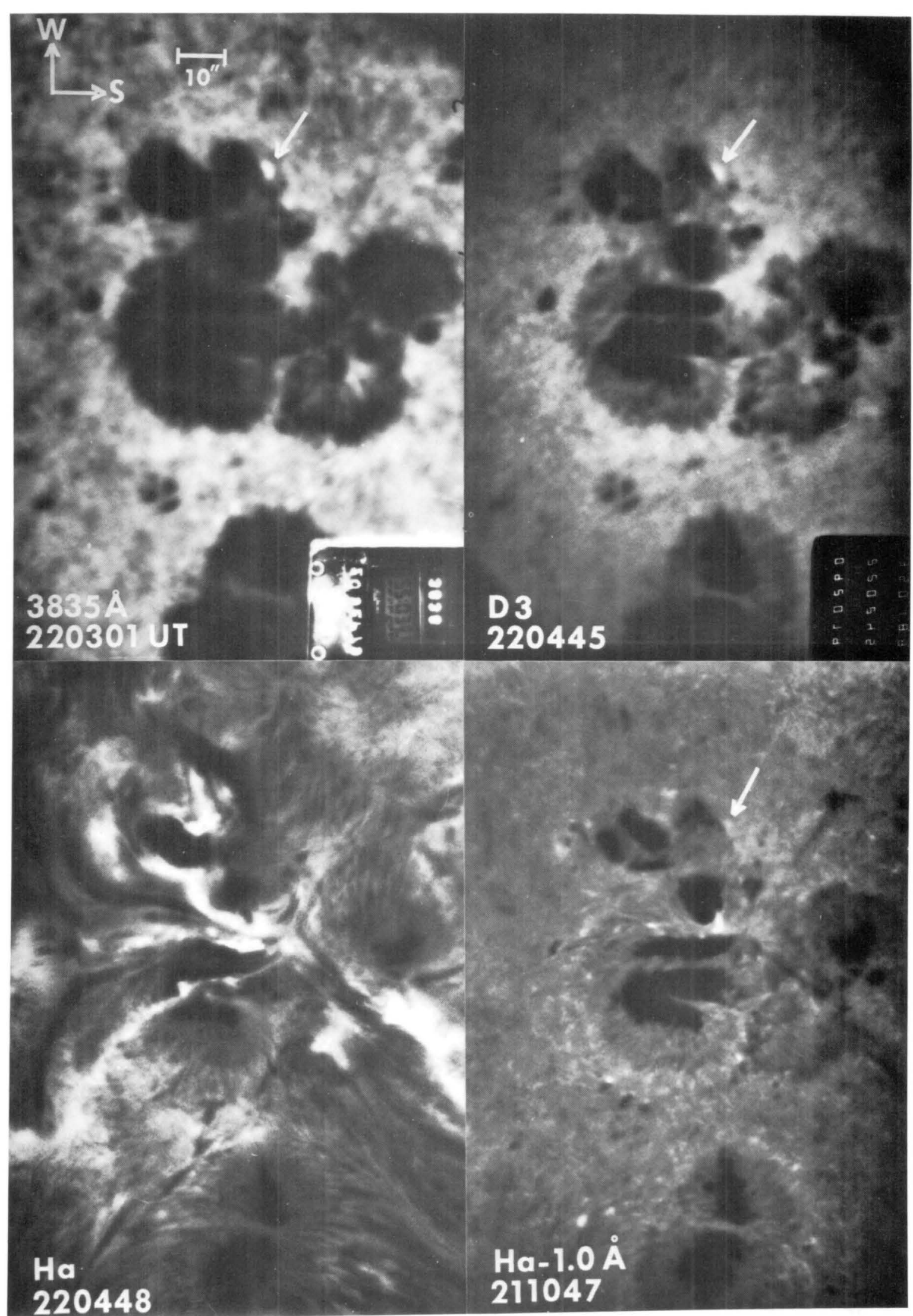


The point was located in or very near the site of many small surges visible in the $\mathrm{H} \alpha$ $-0.7 \AA$ movie. However, the $\mathrm{H} \alpha$ point always remained much less bright than is typical of Ellerman bombs; the brightness at $\mathrm{H} \alpha-0.7 \AA$ can be completely explained by the continuum brightening. A large flare around 18:59 produced brightening over the spot, in fact completely covering it; but the brightening covers many other places as well, so the flare was unrelated to our point.

We measured the intensity of the continuum bright spot by reference to calibrated step wedges developed with the film. The contrast of the spot varies with visibility, hence time variation of brightness is not definite. The intensity at 3835 was $1.2 \times$ photosphere at 22:03:01 UT, and the $\mathrm{D}_{3}$ intensity $16 \mathrm{~s}$ earlier was also $1.2 \times$ photosphere. On a later frame, 22:08:48, the 3835 intensity rose to $1.3 \times$ $\times$ photosphere but the corresponding $\mathrm{D}_{3}$ frames were underexposed. We conclude from these measurements and visual examination that the $D_{3}$ intensity included no line emission, but represented the same continuum as seen in the blue. The films show a roughly parallel development in both colors. If this is so, and the continuum, as measured, is enhanced by roughly the same ratio at both lines, we can conclude that the temperature of the bright point is not much above that of the photosphere. An increase of $250^{\circ}$ would produce an increase of $28 \%$ at 3835 and $18 \%$ at $\mathrm{D}_{3}$; our photometry is satisfactory but the seeing makes it impossible to test for such a color increase, and we cannot rule out some $\mathrm{D}_{3}$ line emission (although the lack of $\mathrm{H} \alpha$ emission makes it unlikely).

What are we seeing? Clearly this phenomenon is related to something below the Sun's surface; the relative absence of $\mathrm{H} \alpha$ emission and the long lifetime and the lack of blue enhancement suggest that the energy comes from below, rather than from above as in a white-light flare. The energy involved in a $20 \%$ increase of the emission from a $2 \times 2.5^{\prime \prime}$ area for $5 \mathrm{hr}$ is prodigious, about $7 \times 10^{30} \mathrm{erg}$, as big as a sizeable flare. The brightening is greater than in the normal network, and probably greater than the penumbral bright points shown by Bray and Loughhead, who quote no numbers; on the other hand their penumbral events occur on a darker background. Above all, this brightening does not look like the penumbral events, nor does it look like anything else we have ever seen. Vorpahl and Pope (1972) have reported on bright points in 3835 associated with emergence of new flux. Our event was associated with changing flux (although not a classic EFR as in their case). It is much brighter in 3835 and much dimmer in offband $\mathrm{H} \alpha$ than the features studied by Vorpahl and Pope. So for these reasons we enter it in the solar zoo, and propose the name penumbra-periphery bright point.

Fig. 1. Frames of the bright point in various wavelengths as marked. An arrow points to the point. The 3835 filter is $60 \AA$ wide and Kodak S0424, a super fine grain film, was used; Kodak S0115 was used for the others. A small flare across another neutral line can be seen on the $\mathrm{H} \alpha-1.0 \AA$ frame. The 3835 and D3 frames were taken with the $65 \mathrm{~cm}$ vacuum Gregorian telescope; the $\mathrm{H} \alpha$ frames, with the $25 \mathrm{~cm}$ vacuum refractor. This photograph was automatically dodged with a Logetronic Printer to reduce artificial large-scale density gradations in the original filtergrams. 


\section{Acknowledgements}

This work was supported by NASA under NGL 05-002-034 and NSF under ATM 76-21132. The observations were made by Eugene Longbrake and Jack Klemroth; we thank Frances Tang and Margaret Liggett for doing the figure.

\section{References}

Bray, R. J. and Loughhead, R. E.: 1964, Sunspots, Plates 3.11-3.13, Chapman and Hall, London. Vorpahl, J. and Pope, T.: 1972, Solar Phys. 25, 347.

Zirin, H.: 1980, Astrophys. J. 235, 618. 